\section{Active mixing during hybridization improves the accuracy and reproducibility of microarray results}

\author{
Christopher J. Schaupp, Guojian Jiang, Timothy G. Myers, and \\ Michael A. Wilson \\ National Institute of Allergy and Infectious Diseases, Bethesda, MD, USA \\ BioTechniques 38:117-119(January 2005)
}

The hybridization process is a critical step that influences the quality of microarray data and contributes to the variability of the resulting expression estimate (1-3). An advantage of the two-color hybridization strategy for custom microarrays is that probe application inefficiencies and other spot-to-spot differences involving identical probes are internally controlled (4). Since the method relies on the relative difference between the two fluorescent signals to estimate the fold change in gene expression, the accuracy and reproducibility of these ratio measurements are fundamental features of a platform's performance. Despite this, gene expression data derived from microarray projects have been reported to underestimate or fail to detect accurate differences in gene expression (5-9).

A conventional hybridization method for custom-printed DNA microarrays is performed by introducing the hybridization solution between the microarray surface and a glass coverslip. With this static hybridization technique, mixing of the hybridization solution is limited by the rate of diffusion and inefficient mixing limits sensitivity since an estimated $0.3 \%$ of the available target solution gains access to the specific probes on the array (1). Active mixing of the liquid-phase target samples is reported to accelerate the kinetics of the hybridization, decrease the amount of time needed to reach equilibrium, and enhance sensitivity $(1,2,10)$. In this study, we compared the performance of a conventional glass coverslip hybridization method to that of the MAUI ${ }^{\circledR}$ hybridization system (BioMicro Systems, Salt Lake City, UT, USA), which performs sample mixing within a sealed, low-volume compartment.

An in vitro culture model was developed to make comparisons between hybridization methodologies on the two-color microarray platform. A reference RNA pool, designated M6, was made by mixing equivalent amounts of total RNA isolated from six different mouse cell lines. Five cell lines (BpRcI, Sp2/0-Ag14, 4T1, RAW264.7, WENI 7.1) obtained from the ATCC (Manassas, VA, USA) originated from a variety of tissue types. A mouse hybridoma line, OKT3, was a gift from Larry Lantz [National Institute of Allergy and Infectious Diseases (NIAID) Custom Antibody Facility, Research Technologies Branch]. OKT3 total RNA served as the test RNA, so that ratios of differential gene expression are modeled by the OKT3/M6 ratios for each messenger RNA (mRNA) transcript. This model system is well suited for comparing the two methods, because it produces thousands of differential ratio measurements over a broad dynamic range. Furthermore, since OKT3 is also a minor component of the pool of six cell lines used for the reference, it improves the precision of measuring ratios because mRNA transcripts present in OKT3 are represented at detectable levels in both channels.

The microarrays used for this study were produced by printing a library of 70-mer oligonucleotide mouse probes (Mouse Genome Oligo Set, version 2.0; Qiagen, Valencia, CA, USA) and 18 control probes from the Spot Report (Ambion, Austin, TX, USA) and Alien ${ }^{\mathrm{TM}}$ Amine (Stratagene, La Jolla, CA, USA) kits onto epoxy-activate glass slides (MWG Biotech, High Point, NC, USA). After printing, the microarrays were kept in a humidified chamber overnight and processed with a series of washes in $0.2 \%$ sodium dodecyl sulfate (SDS), $1 \mathrm{mM} \mathrm{HCl}$, and boiling water. The complete platform description for the microarrays is available in the National Center for Biotechnology Information's (NCBI's) Gene Expression Omnibus under the accession no. GPL1057 (http://www. ncbi.nlm.nih.gov/geo/).

To compare the mixing and static hybridization methods, we produced a pool of M6 cDNA (CyTM5-labeled) and OKT3 cDNA (Cy3-labeled) sufficient for eight replicate hybridizations. The fluorescently labeled cDNA was prepared by reverse transcription with direct incorporation of either Cy3dUTP or Cy5-dUTP (detailed protocol available upon request or online at http://www.niaid.nih.gov/dir/services/ $\mathrm{rtb} / \mathrm{docs} / \mathrm{LABELING.PDF). \quad Four}$ replicate hybridizations were performed for each method, so that cDNA derived from $30 \mu \mathrm{g}$ total RNA per sample was applied to each microarray.

Immediately prior to hybridization, all microarrays were submerged for approximately $30 \mathrm{~min}$ in prehybridization solution $[5 \times$ sodium saline citrate (SSC), 1\% bovine serum albumin (BSA), $0.2 \%$ SDS] maintained at $42^{\circ} \mathrm{C}$ as described previously (11). The slides were washed three times in deionized water, once in isopropyl alcohol, and dried by centrifugation at $50 \times g$ for $3 \mathrm{~min}$. Aliquots of the cDNA stock solution were mixed with a $2 x$ hybridization cocktail so that the final hybridization cocktail consisted of $5 \times$ SSC, $0.2 \%$ SDS, $25 \%$ formamide, with $1 \mu \mathrm{g}$ mouse Cot-1 DNA (Invitrogen, Carlsbad, CA, USA), $4 \mu \mathrm{g}$ yeast transfer RNA (tRNA; Sigma, St. Louis, MO, USA), $1 \mu \mathrm{g}$ poly(dA) (40-60; Amersham Biosciences, Piscataway, NJ, USA). The hybridization cocktails were heated to $98^{\circ} \mathrm{C}$ for $2 \mathrm{~min}$ and centrifuged for $1 \mathrm{~min}$ at $12,000 \times \mathrm{g}$ immediately prior to being applied to the array.

For the static hybridization method, $66 \mu \mathrm{L}$ hybridization cocktail were injected under a $25 \times 60 \mathrm{~mm}$ LifterSlip $^{\mathrm{TM}}$ (Erie Scientific, Portsmouth, NH, USA) so that the solution 
Table 1. Distribution Characteristics of the $\log _{2}$ Ratios Reported by Each Method

\begin{tabular}{|lcccc|}
\hline Hybridization & \multicolumn{3}{c}{ Percentile } & $\begin{array}{c}\text { Interquartile Range } \\
\text { Method }\end{array}$ \\
\cline { 2 - 4 } & $\mathbf{2 5} \%$ & $\mathbf{5 0 \%}$ & $\mathbf{7 5 \%}$ & $\mathbf{( 2 5 \% - 7 5 \% )}$ \\
\hline Static & -0.42 & -0.04 & 0.37 & 0.80 \\
Mixed & -0.65 & -0.03 & 0.51 & 1.16 \\
\hline
\end{tabular}

covered the entire printed area of the array. The slide assemblies were placed inside a closed Tupperware ${ }^{\circledR}$ container that was fitted with a level flat platform that held them above a bed of moistened filter paper. Hybridizations with mixing were performed on the MAUI hybridization system according to the manufacturer's instructions by injecting $40 \mu \mathrm{L}$ hybridization solution into the chamber and sealing the ports. All hybridizations were performed overnight at $45^{\circ} \mathrm{C}$. The slides were washed twice in $2 \times \mathrm{SSC}, 0.2 \% \mathrm{SDS}$, twice in $0.1 \times \mathrm{SSC}$ for $2 \mathrm{~min}$ each wash, and dried by centrifugation at $50 \times g$ for 5 min.

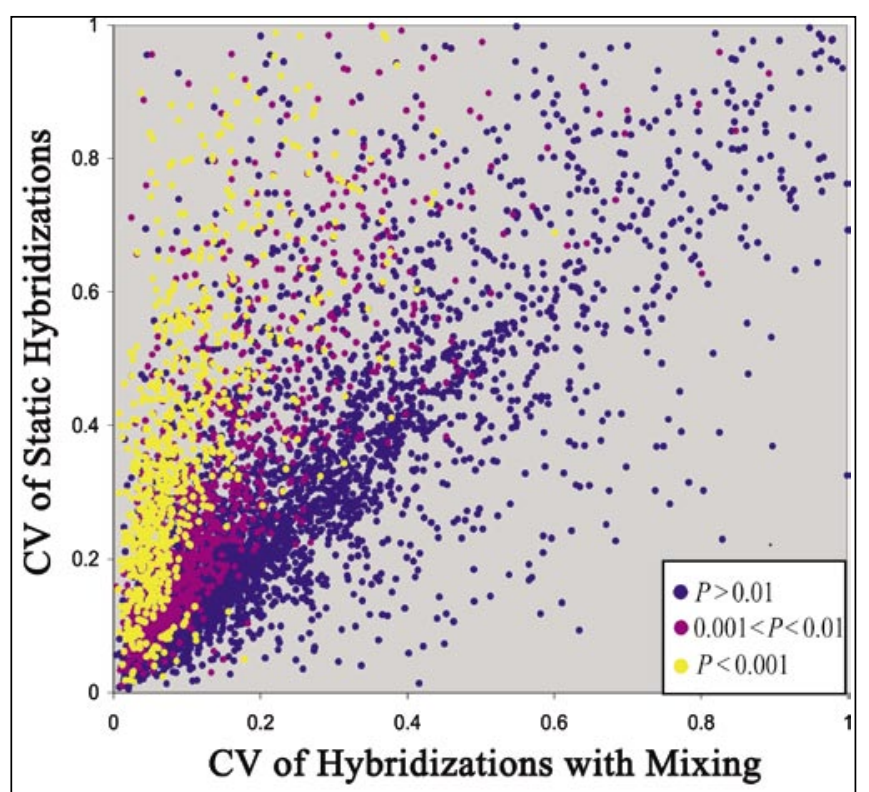

Figure 1. Reproducibility of each hybridization method. The coefficients of variation (CVs) were calculated on replicates for each of the two hybridization methods. The plot compares the cVs for the 6670 mouse features on the arrays that passed the filtering criteria. For each hybridization experiment, spot features were eliminated from further analysis if they were designated "not found" by the GenePix Pro 4.0 software (Axon Instruments, Union City, CA, USA), flagged manually as damaged, or if the signal of either channel was $<2$ SD above the average local background signal. Each experiment was normalized by applying a global factor that set the median ratio of all the analyzed features equal to 1 . For statistical tests, oligonucleotide probes were analyzed if at least three valid measurements were obtained from the four replicates in each group. The cloud of data points above the diagonal indicates that the results of static hybridizations have greater variability for the vast majority of features. Some outliers extend beyond the axes shown.
The average signalto-background ratio of the mixing method was almost 3-fold higher than the static method (not shown). This result is consistent with previously reported comparisons of two similar hybridization methods $(1,2)$. While this increased sensitivity was apparent for the vast we array, the levels of signal associated supplied with the Alien and Spot Report kits) tended to be significantly lower for hybridizations with mixing. Since were of the complementary targets probes should serve as negative controls and surrogates for assessing the contributions of nonspecific signal associated with each hybridization method. Of the 18 negative control probes evaluated, 15 had significantly lower signal in both $\mathrm{Cy} 3$ and Cy5 channels. (Lower signal was defined as significant if the $P$ value for the difference was $P<0.05$ by the Wilcoxon/Kruskal Wallis test.) In contrast, only one probe reported a higher signal for the hybridizations with mixing. From these results we concluded that active mixing was increasing the proportion of sequence-specific targets hybridized to each spot while reducing the component of the signal due to nonspecific factors.

The ratio measurements derived from the mixing hybridization method were more reproducible in general.
A plot comparing the coefficients of variation (CVs) derived from the ratios of replicate hybridizations for each method illustrates a dominant trend toward lower $\mathrm{CV}$ values for the mixed hybridizations (Figure 1). For those features with the significantly different CVs by a paired two-tailed $t$-test $(P<$ $0.01), 96 \%$ reported lower $\mathrm{CV}$ values for the hybridizations that were mixed. We further observed a difference in the distributions of the ratio magnitudes produced by each hybridization method. Table 1 summarizes the distributions of the ratios produced by the two methods, illustrating that the mixing method tended to report ratios over a broader range of values. Since this observation is consistent with previous reports that microarray platforms are vulnerable to reporting compressed gene expression ratios (5-9), we predicted that the hybridizations with mixing were more accurately reporting the true ratios of differential transcript abundances between the two RNA samples.

To assess the accuracy of the measured ratios more directly, we performed spike-in experiments with external, exogenous transcripts from the Spot Report and Alien Amine kits. Prior to cDNA labeling, exogenous transcripts were pooled into two groups and spiked into the two total RNA samples at ratios of 1:2 and 5:1 (Cy3: Cy5). The reported $\log _{2}$ ratios for the mixed hybridizations showed better agreement with the expected 5-fold ratios than the results of the static hybridizations (Figure 2A). In each case, the static hybridization ratios were less than the target ratio, which was consistent with the dampening effect observed for the ratios of total mouse RNA.

To test the effect of reducing the amounts of RNA used in the cDNA labeling reaction, we produced cDNA from 20,10 , and $5 \mu \mathrm{g}$ of the spiked total RNA that had been used in the experiment described above. The accuracy and precision of the results remained consistent over the range of concentrations tested (Figure 2B). This experiment confirmed that the system's accuracy is preserved in the situation that takes practical advantage of the improved sensitivity by labeling 


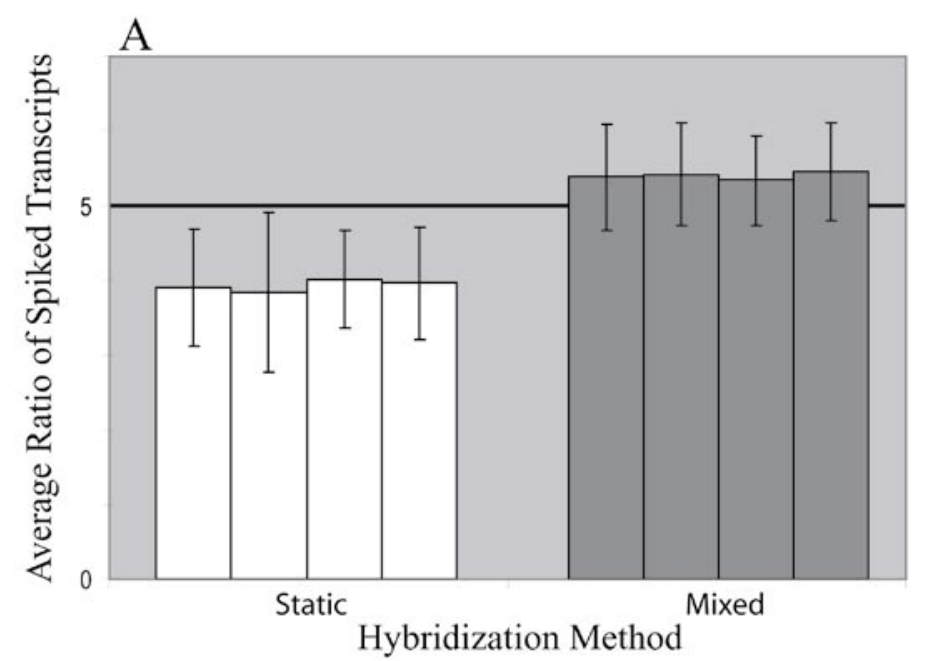

B

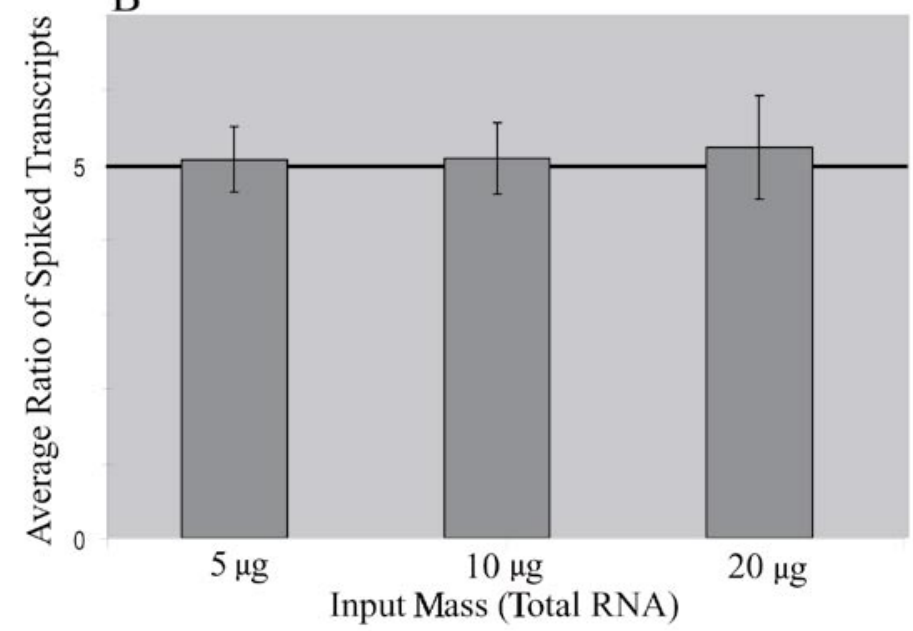

Figure 2. Accuracy of ratios for external controls. Accuracy was assessed by spiking external transcripts into the labeling reaction at known ratios. Two pooled stocks of external transcripts were created by combining transcripts from Stratagene (Pool S) and Ambion (Pool A). Pool S transcripts were spiked into the two different total RNA samples at a 1:2 ratio prior to labeling, and the Pool A transcripts were spiked into the two total RNA samples at a 5:1 ratio. Each total RNA with spikes was taken through a series of serial 2-fold dilutions prior to labeling. The data were standardized on the results of the Pool S transcripts. For each experiment, the normalization factor required to set the Pool S transcripts to the expected average ratio of 0.5 was applied to the set of Pool A transcripts. (A) Data for replicate hybridizations of the static and mixing methods are shown for the Pool A spikes when cDNA derived from $40 \mu \mathrm{g}$ total RNA was hybridized per sample. The mixing hybridization showed better agreement with expected ratio of 5 (horizontal line) than did the static hybridizations. (B) Results of Pool A spikes agreed with the expected ratios when hybridizations with mixing were performed using cDNA derived from 5,10 , and $20 \mu \mathrm{g}$ spiked total RNA.

reduced amounts of total RNA.

Our results show that active mixing of the hybridization solution produced ratio measurements of differential transcript abundance that were more accurate and more reproducible than a conventional static hybridization method. These improvements are likely due to the combined contributions of greater assay sensitivity and reduced nonspecific hybridization. These results confirm that a microarray-based research project will benefit from using a dynamic hybridization system that adequately mixes the sample.

\section{COMPETING INTERESTS STATEMENT}

The authors declare no competing interests.

\section{REFERENCES}

1.Adey, N.B., M. Lei, M.T. Howard, J.D. Jensen, D.A. Mayo, D.L. Butel, S.C. Coffin, T.C. Moyer, et al. 2002. Gains in sensitivity with a device that mixes microarray hybridization solution in a 25-micron-thick chamber Anal. Chem. 74:6413-6417.

2.McQuain, M.K., K. Seale, J. Peek, T.S. Fisher, S. Levy, M.A. Stremler, and F.R. Haselton. 2004. Chaotic mixer improves microarray hybridization. Anal. Biochem. 325:215-226.

3.Sartor, M., J. Schwanekamp, D. Halbleib, I. Mohamed, S. Karyala, M. Medvedovic, and C.R. Tomlinson. 2004. Microarray results improve significantly as hybridization approaches equilibrium. BioTechniques 36:790-796.

4.Brown, P.O. and D. Botstein. 1999. Exploring the new world of the genome with DNA microarrays. Nat. Genet. 21:33-37.

5.Yuen, T., E. Wurmbach, R.L. Pfeffer, B.J. Ebersole, and S.C. Sealfon. 2002. Accuracy and calibration of commercial oligonucleotide and custom cDNA microarrays. Nucleic Acids Res. 30:e48.

6.Barczak, A., M.W. Rodriguez, K. Hanspers, L.L. Koth, Y.C. Tai, B.M. Bolstad, T.P. Speed, and D.J. Erle. 2003. Spotted long oligonucleotide arrays for human gene expression analysis. Genome Res. 13:1775-1785.

7.Iyer, V.R., M.B. Eisen, D.T. Ross, G. Schuler, T. Moore, J.C. Lee, J.M. Trent, L.M. Staudt, et al. 1999. The transcriptional program in the response of human fibroblasts to serum. Science 283:83-87.

8.Korkola, J.E., A.L. Estep, S. Pejavar, S. DeVries, R. Jensen, and F.M. Waldman. 2003. Optimizing stringency for expression microarrays. BioTechniques 35:828-835.

9.Etienne, W., M.H. Meyer, J. Peppers, and R.A. Meyer, Jr. 2004. Comparison of mRNA gene expression by RT-PCR and DNA microarray. BioTechniques 36:618-626.

10.Liu, R.H., R. Lenigk, R.L. Druyor-Sanchez, J. Yang, and P. Grodzinski. 2003. Hybridization enhancement using cavitation microstreaming. Anal. Chem. 75:1911-1917.

11.Hegde, P., R. Qi, K. Abernathy, C. Gay, S. Dharap, R. Gaspard, J.E. Hughes, E. Snesrud, et al. 2000. A concise guide to cDNA microarray analysis. BioTechniques 29:548556.

Received 12 July 2004; accepted 25 August 2004.

Address correspondence to Michael A. Wilson, Ambion Inc., 2130 Woodward Street, Austin, TX 78744, USA. e-mail: mwilson@ambion.com 